\title{
HRTEM Analysis of Au-Cu Nanoparticles
}

\author{
A. Medina-Flores ${ }^{*}$, L. Béjar-Gómez ${ }^{*}$, L. Zamora ${ }^{* *}$, A.L. Medina-Almazán ${ }^{* *}$, J.L. Bernal ${ }^{* * *}$ \\ *Instituto de Investigaciones Metalúrgicas, UMSNH, Edificio U, ciudad Universitaria, Morelia, \\ Michoacán. 58000. México. \\ ${ }^{* *}$ ININ. Carretera México-Toluca s/n. Ocoyoacác. 52750. México. \\ *** Universidad Politécnica del Valle de México. Avenida Mexiquense s/n. C.P. 54910. Tultitlán \\ Estado de México.
}

In recent years, the synthesis of bimetallic nanoparticles has been very successful in obtaining small particles of multiple elements [1-3] followed by analysis of their properties well supported by analytical methods [4]. In the emerging field of new materials, designing, synthesis, and atomistic characterization of nanostructures have become very important, because the manipulation of structure has a direct effect in their macroscopic properties. In fact, the physical and chemical properties of nanoparticles are directly related to their chemical composition, size and volume, and surface structural characteristics. Particularly, the case of AuCu nanoparticles has attracted vast attention because of the possibility of using them in catalysis and other fields.

The synthesis of bimetallic particles was made sol-gel method. Structural characterization of the samples was made using a FEG TEM TECNAI F20 microscope with analytical equipments attached. The samples were prepared by spreading a droplet of colloidal solution of $\mathrm{AuCu}$ nanoparticles onto a carbon film supported by a $\mathrm{Cu}$ grid and subsequent drying in vacuum. The samples were analyzed by high resolution microscopy and the elemental composition. The high resolution electron microscopy (HREM) images were processed by digital methods in the real and Fourier spaces. In Figure 1, show a truncated octahedron cluster with like fcc structure. The shape and contrast are quite similar to the single element nanoparticles and the distances show a homogeneous distribution of values between both the $\mathrm{Au}$ and $\mathrm{Cu}$, while just small differences are observed near to the edge of the particles. This behavior was expected as the difference in lattice constants between the two metals is small. However, obtaining direct evidences of the elemental composition variations are not easy. It is likely that both elements are in the observed region as can see in the figure 2.

\section{References}

[1] De Meijer RJ and et al. Nanostruct Mater 8, 1997. 205-213.

[2] Link S and et al. J Chem Phys 11, 1999. 1255-1262.

[3] Ruiz A and et al. Mater Sci Eng C 19, 2002. 105-109

[4] Jose'-Yacamán M and et al. Top Catal 18, 2002. 167-173. 

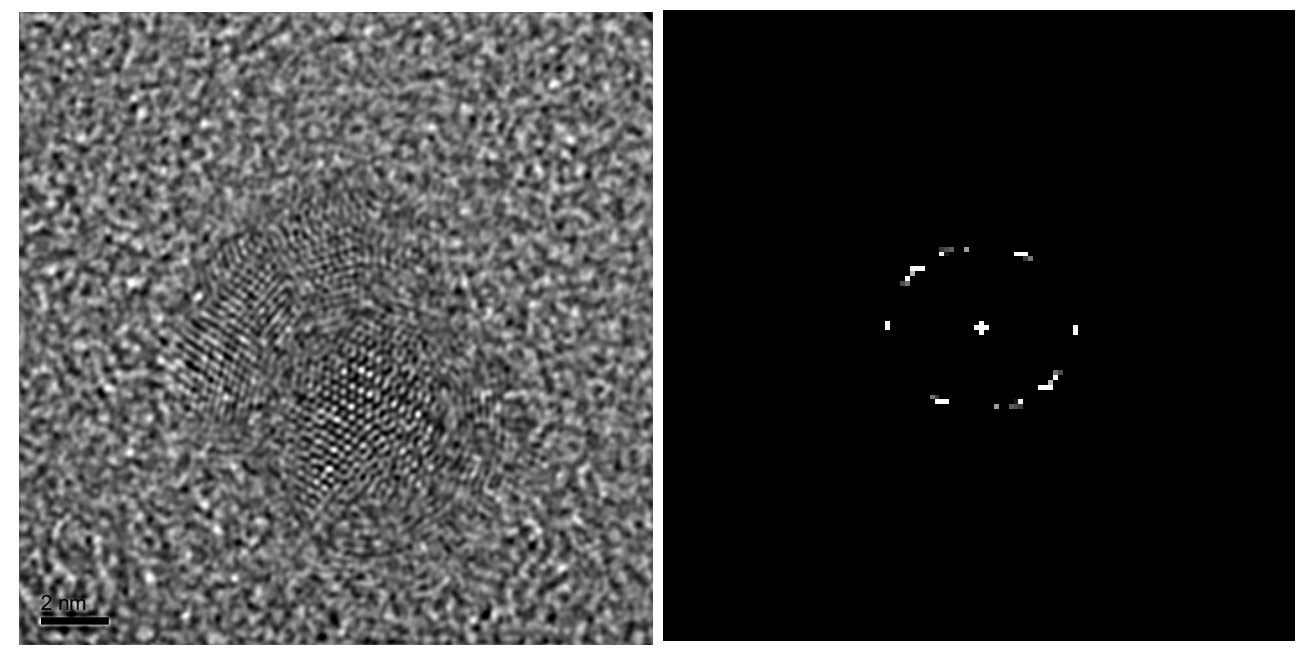

Figure 1: HRTEM image of a fcc-like structure with its corresponding FFT pattern.

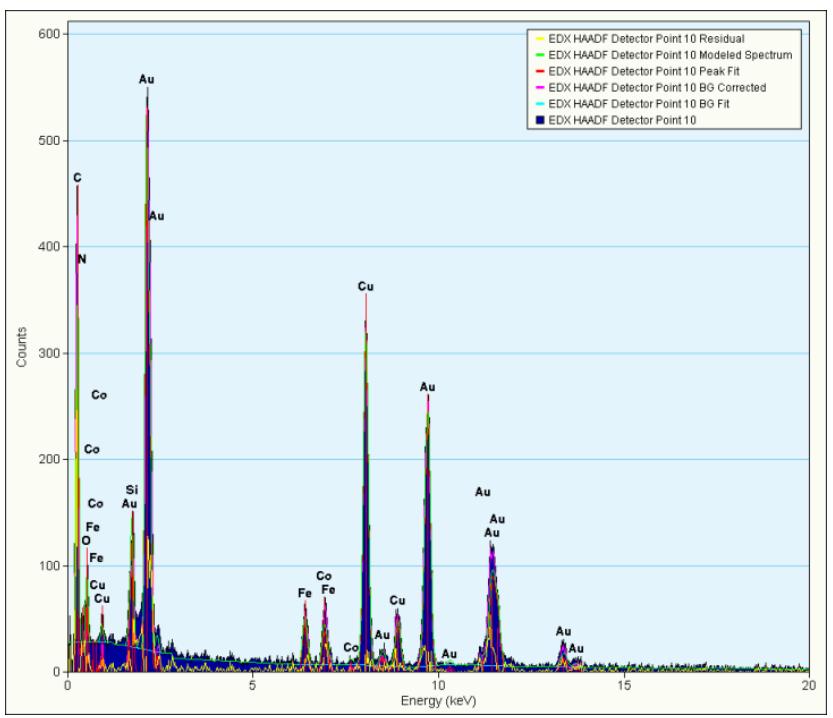

Figure 2: EDS spectrum of the $\mathrm{AuCu}$ nanoparticle 